\title{
DIFFERENCE SEQUENCE SPACES AND MATRIX TRANSFORMATIONS
}

\author{
Geeta Bhakta Joshi, Ph.D. \\ Prof. Mathematics Department, Pulchowk Campus, Institute of Engineering, Tribhuvan University \\ Shailendra Kumar Mishra, Ph.D. \\ Prof. Mathematics Department, Pulchowk Campus, Institute of Engineering, Tribhuvan University
}

Shankar Rajpant, Ph.D.

Ex-Chairman, Prof. Central Department of Mathematics, Tribhuvan University, Kirtipur

\begin{abstract}
In this paper, we extend the work of Gaur, A.K. and Mursaleen [6] and also extend the work of Mursaleen, Gaur, A.K. and Saifi, A.H.[16]. We characterize the matrices that $\operatorname{map} S_{r}(p, \Delta), \Delta \lambda_{\infty}(p), \Delta c_{0}(p), \Delta c(p)$ and $\lambda_{\infty}\left(\Delta_{r} p\right)$ into $\Omega(t)$.
\end{abstract}

\section{INTRODUCTION}

Let $\lambda_{\infty}, c$ and $c_{0}$ be the sets of all bounded, convergent and null sequences of $x=\left(x_{k}\right)$ respectively. Let $\omega$ denote the set of all complex sequences and let $\lambda_{1}$ denote the set of all convergent and absolutely convergent series.

If $p=\left(p_{k}\right)$ is a bounded sequence of strictly positive real numbers, and if $\Delta x=\left(x_{k}-x_{k-1}\right)$, then we have

$$
\begin{gathered}
\lambda(p)=\left\{x=\left(x_{k}\right): \sum_{k}\left|x_{k}\right|^{p_{k}}<\infty\right\} ; \\
\lambda_{\infty}(p)=\left\{x=\left(x_{k}\right) \in \omega: \sup _{k}\left|x_{k}\right|^{p_{k}}<\infty\right\} ; \\
\Delta \lambda_{\infty}(p)=\left\{x=\left(x_{k}\right): \Delta x \in \lambda_{\infty}(p)\right\} ; \\
\Delta c(p)=\left\{x=\left(x_{k}\right): \Delta x \in c(p)\right\} ; \\
\Delta c_{0}(p)=\left\{x=\left(x_{k}\right): \Delta x \in c_{0}(p)\right\}
\end{gathered}
$$

If all the terms of $p=\left(p_{k}\right)$ are constant and $p>0$, then $\Delta \lambda_{\infty}(p)=\Delta \lambda_{\infty}$,
$\Delta c(p)=\Delta c \quad$ and $\quad \Delta c_{0}(p)=\Delta c_{0} . \quad$ The classes $\Delta \lambda_{\infty}, \Delta c, \Delta c_{0}$ are normed spaces under the norm

$\|x\|=\|\Delta x\|_{\infty}$

where $\|\cdot\|_{\infty}$ is the usual norm on $\lambda_{\infty}, c$ or $c_{0}$. It is known that if $\left(p_{k}\right) \in \lambda_{\infty}$ then $\Delta c_{0}(p)$ is a paranormed space paranormed by $g^{*}(x)=g(\Delta x) ; \Delta \lambda_{\infty}(p)$ and $\Delta c(p)$ are paranormed by $g^{*}(x)=g(\Delta x)$ if and only if inf $p_{k}>0$, where $g$ is the usual paranorm on $\lambda_{\infty}(p), c(p)$ and $c_{0}(p)$.

Let $z$ be any sequence and $Y$ be any subset of $\omega$. Then

$$
z^{-1} . Y=\left\{x \in \omega: z x=\left(z_{k} x_{k}\right)_{1}^{\infty} \in Y\right\}
$$

For any subset $X$ of $\omega$, the sets

$$
X^{\alpha}=\underset{x \in X}{\mathrm{I}}\left(x^{-1} \cdot \lambda_{1}\right) \text { and } X^{\beta}=\operatorname{I}_{x \in X}\left(x^{-1} \cdot c s\right)
$$

are called the $\alpha^{-}$and $\beta^{-}$duals of $X$. 
We define the linear operators $\Delta, \Delta^{-1}: \omega \rightarrow \omega$ by $\Delta x=\left(\Delta x_{k}\right)_{1}^{\infty}=\left(x_{k}-x_{k+1}\right)_{1}^{\infty}$,

and

$\Delta^{-1} x=\left(\Delta^{-1} x_{k}\right)_{1}^{\infty}=\left(\sum_{j=1}^{k-1} x_{j}\right)_{1}^{\infty}$,

$\Delta^{-1} x=0$.

Let,

$S_{r}(\Delta)=\left\{x \in \omega:\left(k^{r}\left|\Delta x_{k}\right|\right)_{k=1}^{\infty} \in c_{0}\right\}$

Let $p=\left(p_{k}\right)_{1}^{\infty}$ be an arbitrary sequence of positive reals and $r \geq 1$, then Gaur, A.K. and Mursaleen [6] have defined a new sequence space

$S_{r}(p, \Delta)=\left\{x \in \omega:\left(k^{r} \Delta x_{k}\right)_{k=1}^{\infty} \in c_{0}(p)\right\}$

where

$c_{0}(p)=\left\{x=\left(x_{k}\right) \in \omega: \lim _{k \rightarrow \infty}\left|x_{k}\right|^{p_{k}}=0\right\}$

If $p=e=(1,1,1, \mathrm{~K} \mathrm{~K})$, then the set $S_{r}(p, \Delta)$ reduces to the set $S_{r}(\Delta)$. For $r=0, S_{r}(p, \Delta)$ is the same as $\Delta c_{0}(p)$. In

[16] Mursaleen, Gaur, A.K. and Saif, A.H. has defined

$\lambda_{\infty}\left(\Delta_{r} p\right)=\left\{x=\left(x_{k}\right): \Delta_{r} x \in \lambda_{\infty}(p), r<1\right\}$

where

$\Delta_{r} x=\left(k^{r} \Delta x_{k}\right)_{1}^{\infty}$.

\subsection{MATRIX TRANSFORMATIONS}

For any infinite complex matrix $A=\left(a_{n k}\right)_{n, k=1}^{\infty}$, we write $A=\left(a_{n k}\right)$ for the sequence in the $n^{\text {th }}$ row of c. Let $X$ and $Y$ be two subsets of $\omega$. By $(X, Y)$ we denote the class of all matrices of $A$ such that the series $A_{n}(x)=\sum_{k=1}^{\infty} a_{n x} x_{k}$ converges for all $x \in X$ and $n \in N$, and the sequence $A x=\left(A_{n}(x)\right)_{n=1}^{\infty} \in Y$ for all $x \in X$.

Fricke and Fridy [8] introduced a new sequence space $\Omega(t)$. We shall give the definition of $\Omega(t)$ and some results from [8].

For each $r=\left(r^{k}\right)$ in the interval $(0,1)$ let

$G(r)=\left\{x=\left(x_{k}\right) \in \omega: x_{k}=O\left(t_{k}\right)\right\}$.

We define the set of geometrically sequences as

$G={\underset{r \in(0,1)}{\mathrm{Y}}} G(r)$

The analytic sequences are defined by

$\mathrm{A}=\left\{x=\left(x_{k}\right) \in \omega: \limsup _{n}\left|x_{n}\right|^{\frac{1}{n}}<\infty\right\}$

Obviously $G \subseteq$ A

In [8] , Fricke and Fridy replaced the geometric sequence $\left(r^{k}\right)$ with a nonnegative number sequence $t$ and defined.

$\Omega(t)=\left\{x=\left(x_{k}\right) \in \omega: x_{k}=O\left(t_{k}\right)\right\}$.

Here for a given matrix $A$, the sequence $\sigma=\left(\sigma_{n}\right)_{n=1}^{\infty}$ is defined by

$\sigma_{n}=\sum_{k=0}^{\infty}\left|a_{n k}\right|$

Corollary 1.2.1. ( see [8], Corollary 2B) : If $A$ is an infinite matrix and $t$ is nonnegative number sequence, then $A$ maps $\lambda_{\infty}, c, c_{0}$ into $\Omega(t)$ if and only if $\sigma \in \Omega(t)$.

Corollary 1.2.2. (see [16], Theorem 2.1. and Theorem 3.1.): $A \in\left(X, Y\left(\Delta_{r}\right)\right)$ if and only if

(i) $A_{1} \in X^{\beta}$ where,

$$
\begin{aligned}
& X^{\beta}=D_{2}(p) \\
& ={ }_{N \geq 2}\left\{a \in \omega: \sum_{k=1}^{\infty} k^{-r} a_{k} \sum_{j=1}^{k-1} N^{\frac{1}{p}} \text { converges }, \sum_{k=1}^{\infty} k^{-r} N^{\frac{1}{p_{k}}}\left|R_{k}\right|<\infty\right\}, \\
& R_{k}=\sum_{m=k+1}^{\infty} a_{m} \\
& \text { (ii) } B \in(X, Y), \\
& \text { where } B=\left(b_{n k}\right) \text { is defined by } b_{n k} \\
& =k^{r}\left(a_{n k}-a_{n+1, k}\right) \text { for } r<1 \text { and } n, k=1,2, \mathrm{~K}
\end{aligned}
$$


Remark 1.2.1. If one wishes to have a matrix $A$ that transforms every null sequence into a sequence that conveges at least as rapidly as some $t_{n} \downarrow 0$, thus $A$ must satisfy $\sigma \in \Omega(t)$. Similarly, if $t$ is a nonzero constant sequence, then $\Omega(t)=\lambda_{\infty}$, and in this case Corollary 1.2.1.reduces to the well known result that $A$ preserves boundedness if and only if $\sigma$ is bounded.

Remark 1.2.2. This remark is about obtaining a "given rate of convergence" by mapping $c_{0}$ into $\Omega(t)$. The work [4,9] has shown that regular matrices can not accelerate the rate of convergence of every null sequences. Therefore we emphasize that having $A$ map $c_{0}$ into $\Omega(t)$ does not say that every sequence in $c_{0}$ is accelerated, even if $t_{n} \downarrow 0$ vary rapidly; some sequences that are already in $\Omega(t)$ may map into other members of $\Omega(t)$ that converge at same rate or slower.

Now we characterize the matrices that map $S_{r}(p, \Delta), \quad \Delta \lambda_{\infty}(p), \quad \Delta c_{0}(p)$, $\Delta c(p)$ and $\lambda_{\infty}\left(\Delta_{r} p\right)$ into $\Omega(t)$.

Theorem 1.2.1. $A \in\left(S_{r}(p, \Delta), \Omega(t)\right)$ if and only if

$$
\left(\sum_{k=1}^{\infty}\left|a_{n k}\right| \frac{N^{\frac{-1}{p_{k}}}}{k^{r}}\right) \in \Omega(t) \text { for some }
$$

integer $N>1$.

$$
R \in\left(S_{r}(p, \Delta), \Omega(t)\right)
$$

where, $R=\left(r_{n k}\right)=\left(\sum_{v=k}^{\infty} a_{n v}\right)$.

Proof. If $A \in\left(S_{r}(p, \Delta), \Omega(t)\right)$ then the series $\sum_{k=1}^{\infty} a_{n k} x_{k} \quad$ is convergent and $\left(A_{n}(x)\right)_{n=1}^{\infty} \in \Omega(t)$ for each $n \in N$ and $x \in\left(S_{r}(p, \Delta)\right)$.
In order to see that the condition ( $\mathrm{i}$ ) is necessary, we assume that for some $N>1$,

$$
\left(\sum_{k=1}^{\infty}\left|a_{n k}\right| \frac{N^{\frac{-1}{p_{k}}}}{k^{r}}\right) \notin \Omega(t)
$$

Let the matrix $\mathrm{C}$ be defined by

$$
\left(C=\left(C_{n k}\right)=\left(a_{n k} \frac{N^{\frac{-1}{p_{k}}}}{k^{r}}\right)\right)
$$

Then from corollary 1.2.1, it follows that $C \notin\left(c_{0}, \Omega(t)\right)$. But as, $S_{r}(p, \Delta)=\left\{x \in \omega:\left(k^{r} \Delta x_{k}\right)_{1}^{\infty} \in c_{0}(p)\right\}$, $C \notin\left(S_{r}(p, \Delta), \Omega(t)\right)$. Hence there is a sequence $\quad x \in c_{0} \quad$ such that $\sum_{k=1}^{\infty} C_{n k} x_{k} \neq O(1)$.

We now define a sequence $v=\left(v_{k}\right)$ by $v_{k}=\frac{N^{\frac{-1}{p_{k}}}}{k^{r}} x_{k}$;

so that $v_{k}\left(k^{r} N^{\frac{1}{p_{k}}}\right)=x_{k}$. Then $v \in S_{r}(p, \Delta)$ and

$\sum_{k=1}^{\infty} a_{n k} v_{k}=\sum_{k=1}^{\infty} C_{n k} x_{k} \neq O(1)$

This contradicts that

$A \in\left(S_{r}(p, \Delta), \Omega(t)\right)$.

Thus the condition ( $\mathrm{i}$ ) is necessary.

In order to prove that the condition ( ii ) is necessary we assume that ( ii ) is false. Then there is a sequence $x=\left(x_{v}\right) \in S_{r}(p, \Delta)$ with $\left|k^{r} \Delta x_{k}\right|=1$ such that

$\sum_{v=1}^{\infty} r_{n v} x_{v} \neq O(1)$ 
We now define a sequence $y=\left(y_{k}\right)$ by

$$
y_{v}=\sum_{i=1}^{v} x_{i}
$$

Then $y \in S_{r}(p, \Delta)$ and

$$
\sum_{v=1}^{\infty} a_{n v} y_{v}=\sum_{v=1}^{\infty} r_{n v} x_{v} \neq O(1)
$$

This contradicts the fact that $A \in\left(S_{r}(p, \Delta), \Omega(t)\right)$. Thus the condition ( ii ) is necessary.

We now prove the sufficiency part of the theorem. Suppose that the given condition of the theorem is satisfied. Then there exists a $\mu>0$ such that

$$
\left(\sum_{k=1}^{\infty}\left|a_{n k}\right| \frac{N^{\frac{-1}{p_{k}}}}{k^{r}}\right) \leq \mu t^{n} \text {, for each } n \in N .
$$

Let $\quad x \in S_{r}(p, \Delta) . \quad$ Then $\left(k^{r}\left|\Delta x_{k}\right|\right)<\frac{1}{N^{p_{k}}}$, for sufficiently large value of $k$.. Now we write,

$$
A_{n}(m, x)=\sum_{k=1}^{\infty} a_{n k} x_{k}=\sum_{k=1}^{m} r_{n k} \Delta x_{k}-r_{n+1, m} \sum_{k=1}^{m} \Delta x_{k}, m \in N
$$

Since,

$$
\begin{aligned}
& \sum_{k=1}^{\infty}\left|r_{n k}\right|\left|\Delta x_{k}\right| \leq \sum_{k=1}^{\infty}\left|r_{n k}\right| \frac{1}{k^{r} N^{\frac{1}{p_{k}}}} \text {, where } \\
& \sum_{k=1}^{\infty}\left|r_{n k}\right| \frac{1}{k^{r} N^{\frac{1}{p_{k}}}} \in \Omega(t) \\
& \leq \mu t^{n}, \text { for each } n \in N .
\end{aligned}
$$

Therefore the convergence of

$$
\sum_{k=1}^{\infty}\left|a_{n k}\right| \frac{1}{k^{r} N^{\frac{1}{p_{k}}}}
$$

implies that

$$
r_{n+1, m} \sum_{k=1}^{m} \frac{1}{k^{r} N^{\frac{1}{p_{k}}}}=o(1)
$$

Hence,

$$
A_{n}(x)=\sum_{k=1}^{\infty} a_{n k} x_{k}=\sum_{k=1}^{\infty} r_{n k} \Delta x_{k}
$$

Since $\quad x \in S_{r}(p, \Delta)$ if and only if $\Delta_{r} x \in c_{0}(p)$, where $\Delta_{r} x=\left(k^{r} \Delta x_{k}\right)$. Therefore by condition ( ii ) it follows that $A_{n}(x)$ exists for each $x \in S_{r}(p, \Delta)$ and $A x \in \Omega(t)$. Thus $A \in\left(S_{r}(p, \Delta), \Omega(t)\right)$.

Theorem 1.2.2. $A \in\left(\Delta \lambda_{\infty}(p), \Omega(t)\right)$ if and only if

$$
\left(A_{n}\left(\sum_{m=1}^{k} N^{\frac{1}{p_{m}}}\right)\right)_{n=1}^{\infty} \in c, N>1 ;
$$

(ii ) $\quad R \in\left(\lambda_{\infty}(p), \Omega(t)\right)$, where $R=\left(r_{n k}\right)=\left(\sum_{v=k}^{\infty} a_{n v}\right)$.

Proof. If $A \in\left(\Delta \lambda_{\infty}(p), \Omega(t)\right)$ then the series $\sum_{k=1}^{\infty} a_{n k} x_{k} \quad$ is convergent and $A_{n}(x) \in \Omega(t) \quad$ for $\quad$ some $\quad n \in N \quad$ and $x \in \Delta \lambda_{\infty}(p)$. Since,

$x=\left(\sum_{m=1}^{k} N^{\frac{1}{p_{m}}}\right)_{k=1}^{\infty} \in \Delta \lambda_{\infty}(p)$

Then it follows that

$$
\sum_{k=1}^{\infty} a_{n k}\left(\sum_{m=1}^{k} N^{\frac{1}{p_{m}}}\right)
$$

converges for each $n \in N$. Therefore (i) is necessary.

In order to see that the condition ( ii ) is necessary, we assume that (ii) is false. Then 
there is a sequence $x=\left(x_{v}\right) \in \lambda_{\infty}(p)$ with $\sup _{k}\left|x_{v}\right|^{p_{k}}=1 \quad$ such that

$$
\sum_{v=1}^{\infty} r_{n v} x_{v} \neq O(1)
$$

We now define a sequence $y=\left(y_{v}\right)$ by $y_{v}=\sum_{i=1}^{v} x_{i}$

Then $\quad y \in \Delta \lambda_{\infty}(p) \quad$ and $\sum_{v=1}^{\infty} a_{n v} y_{v}=\sum_{v=1}^{\infty} r_{n v} x_{v} \neq O(1)$.

This contradicts that $A \in\left(\Delta \lambda_{\infty}(p), \Omega(t)\right)$. Thus the condition ( ii ) is also necessary.

We now prove the sufficiency part of the theorem. Suppose that the given conditions of the theorem are satisfied. Let $x \in \Delta \lambda_{\infty}(p)$. Then there is an integer $N>\max \left(1, \sup _{k}\left|\Delta x_{k}\right|^{p_{k}}\right)$.

Now we write,

$A_{n}(m, x)=\sum_{k=1}^{\infty} a_{n k} x_{k}=\sum_{k=1}^{m} r_{n k} \Delta x_{k}-r_{n+1, m} \sum_{k=1}^{m} \Delta x_{k}, m \in N$

Since,

$\sum_{k=1}^{\infty}\left|r_{n k}\right|\left|\Delta x_{k}\right| \leq \sum_{k=1}^{\infty}\left|r_{n k}\right| N^{\frac{1}{p_{k}}}$

and

$$
\left(\sum_{k=1}^{\infty}\left|r_{n k}\right| N^{\frac{1}{p_{k}}}\right)_{n=1}^{\infty} \in \Omega(t)
$$

Therefore the convergence of

$$
\sum_{k=1}^{\infty} a_{n k}\left(\sum_{i=1}^{k} N^{\frac{1}{p_{i}}}\right)
$$

implies that

$$
r_{n+1, m} \sum_{k=1}^{m} N^{\frac{1}{p_{k}}}=o(1)
$$

Hence,

$$
A_{n}(x)=\sum_{k=1}^{\infty} a_{n k} x_{k}=\sum_{k=1}^{\infty} r_{n k} \Delta x_{k}
$$

Since, $x \in \Delta \lambda_{\infty}(p)$ if and only if $\Delta x \in \lambda_{\infty}(p)$. Therefore by condition ( ii ) it follows that $A_{n}(x)$ exists for each $x \in \Delta \lambda_{\infty}(p) \quad$ and $\quad A x \in \Omega(t)$. Thus, $A \in\left(\Delta \lambda_{\infty}(p), \Omega(t)\right)$.

Theorem 1.2.3. Let $\left(p_{k}\right) \in \lambda_{\infty}$. Then $A \in\left(\Delta c_{0}(p), \Omega(t)\right)$ if and only if

$$
\left(A_{n}\left(\sum_{m=1}^{k} N^{\frac{-1}{p_{m}}}\right)\right)_{n=1}^{\infty} \in c, \quad N>1 ;
$$

(ii ) $\quad R \in\left(c_{0}(p), \Omega(t)\right)$ with $R$ as above.

This follows from the arguments given in Theorem ( 1.2.2) and [pp.80, 13].

Theorem 1.2.4. Let $\left(p_{k}\right) \in \lambda_{\infty}$. Then $A \in(\Delta c(p), \Omega(t))$ if and only if (i) $\quad A \in\left(\Delta c_{0}(p), \Omega(t)\right)$;

(ii ) $\left(\sum_{k=1}^{\infty} k a_{n k}\right)_{n=1}^{\infty} \in \Omega(t)$

This follows from Theorem ( 1.2.3) and [pp.80, 15].

Theorem 1.2.5. $A \in\left(\lambda_{\infty}\left(\Delta_{r} p\right), \Omega(t)\right)$ if and only if

( i ) $\quad\left(\sum_{k=1}^{\infty}\left|a_{n k}\right| k^{-r} N^{\frac{1}{p_{k}}}\right) \in \Omega(t)$ for every integer $N>1$.

(ii ) $\quad R \in\left(\lambda_{\infty}\left(\Delta_{r} p\right), \Omega(t)\right)$

where $R=\left(r_{n k}\right)=\left(\sum_{v=k}^{\infty} a_{n v}\right)$ 
Proof. Let us assume that $A \in\left(\lambda_{\infty}\left(\Delta_{r} p\right), \Omega(t)\right) \quad$ but $\left(\sum_{k=1}^{\infty}\left|a_{n k}\right| k^{-r} N^{\frac{1}{p_{k}}}\right)_{n=1}^{\infty} \notin \Omega(t)$ for every integer $N>1$. Then from corollary 1.2.1 and [8], it follows that the matrix

$B=\left(b_{n k}\right)=\left(a_{n k} k^{-r} N^{\frac{1}{p_{k}}}\right) \notin\left(\lambda_{\infty}\left(\Delta_{r}\right), \Omega(t)\right)$

Therefore there exists an $x \in \lambda_{\infty}\left(\Delta_{r}\right)$ with $\sup _{k}\left|x_{k}\right|=1$ such that

$\sum_{k=1}^{\infty} a_{n k} k^{-r} N^{\frac{1}{p_{k}}} x_{k} \neq O(1)$.

Now define a sequence $u=\left(u_{k}\right)$ by $u_{k}=\sum_{i=1}^{k} k^{-r} N^{\frac{1}{p_{i}}} x_{i}$

It is clear that $u \in \lambda_{\infty}\left(\Delta_{r} p\right)$ and

$\sum_{k=1}^{\infty} a_{n k} u_{k}=\sum_{k=1}^{\infty} a_{n k} k^{-r} N^{\frac{1}{p_{k}}} x_{k} \neq O(1)$.

This contradicts the fact that $A \in\left(\lambda_{\infty}\left(\Delta_{r} p\right), \Omega(t)\right)$.

Hence, we must have,

$$
\left(\sum_{k=1}^{\infty}\left|a_{n k}\right| k^{-r} N^{\frac{1}{p_{k}}}\right)_{n=1}^{\infty} \in \Omega(t) .
$$

In order to see that the condition ( ii ) is necessary let us assume that (ii ) is false. Then there exists a sequence $x=\left(x_{v}\right) \in \lambda_{\infty}\left(\Delta_{r} p\right) \quad$ with $\sup _{v}\left|x_{v}\right|^{p_{v}}=1 \quad$ i.e. $\sup _{k}\left|k^{r} \Delta x_{k}\right|^{p_{k}}=1$

such that

$$
\sum_{v=1}^{\infty} r_{n v} x_{v} \neq O(1)
$$

We now define a sequence $y=\left(y_{v}\right)$ by

$$
\begin{aligned}
y_{v}= & \sum_{i=1}^{v} x_{i} . \\
& \text { Then } y \in \lambda_{\infty}\left(\Delta_{r} p\right) \text { and }
\end{aligned}
$$

$$
\sum_{v=1}^{\infty} a_{n v} y_{v}=\sum_{v=1}^{\infty} r_{n v} x_{v} \neq O(1)
$$

This contradicts the fact that $A \in\left(\lambda_{\infty}\left(\Delta_{r} p\right), \Omega(t)\right)$. Thus the condition (ii) is necessary.

Next, suppose that the given conditions are satisfied. Then there exists a constant $M>0$ such that $\sum_{k=1}^{\infty}\left|a_{n k}\right| k^{-r} N^{\frac{1}{p_{k}}} \leq M t_{n}$, for each $n \in N$.

Let $x \in \lambda_{\infty}\left(\Delta_{r} p\right)$. Then there is a positive number $N>\max \left(1, \sup _{k}\left|x_{k}\right|^{p_{k}}\right)$

Now we write,

$A_{n}(m, x)=\sum_{k=1}^{\infty} a_{n k} x_{k}=\sum_{k=1}^{m} r_{n k} \Delta x_{k}-r_{n+1, m} \sum_{k=1}^{m} \Delta x_{k}, m \in N$

Since,

$$
\begin{aligned}
& \sum_{k=1}^{\infty}\left|r_{n k}\right|\left|\Delta x_{k}\right| \leq \sum_{k=1}^{\infty}\left|r_{n k}\right| k^{-r} N^{\frac{1}{p_{k}}} \text {, where } \\
& \sum_{k=1}^{\infty}\left|r_{n k}\right| k^{-r} N^{\frac{1}{p_{k}}} \in \Omega(t) \\
& \leq \mu t^{n}, \text { for each }
\end{aligned}
$$

$n \in N$.

Therefore the convergence of

$\sum_{k=1}^{\infty}\left|a_{n k}\right| k^{-r} N^{\frac{1}{p_{k}}}$

implies that 


$$
r_{n+1, m} \sum_{k=1}^{m} k^{-r} N^{\frac{1}{p_{k}}}=o(1)
$$

Hence,

$$
\begin{aligned}
& A_{n}(x)=\sum_{k=1}^{\infty} a_{n k} x_{k}=\sum_{k=1}^{\infty} r_{n k} \Delta x_{k} \\
& \text { Since } \quad x \in \lambda_{\infty}\left(\Delta_{r} p\right) \text { if and only }
\end{aligned}
$$
if $\Delta_{r} x \in \lambda_{\infty}(p)$. Therefore by condition ( ii ) it follows that $A_{n}(x)$ exists for each $x \in \lambda_{\infty}\left(\Delta_{r} p\right)$ and $A_{n}(x)=O\left(t_{n}\right)$. Then $A x \in \Omega(t) \quad$ for $\quad$ arbitrary $x \in \lambda_{\infty}\left(\Delta_{r} p\right)$. Thus $A \in\left(\lambda_{\infty}\left(\Delta_{r} p\right), \Omega(t)\right)$.

\section{REFERENCES}

[1] Ahmad, Z.U. and Mursaleen, Köthe Toeplitz duals of some new sequence spaces and their matrix maps., Publ. Inst. Math.(Beograd) 42 (56) (1987), 57-61.

[2] Choudhary, B. and Nanda, S., Functional Analysis with Applications, Wiley Eastern Limited, 1989.

[3] Choudhary, B. and Mishra, S.K., A note on certain sequence spaces, J. Analysis 1 ( 1993 ), 139-148.

[4] Delahaya,J.P., and Germain-Bonne, The set of logarithmically convergent sequences can not be accelerated, SIAM J .Numer. Anal. 19 (1982), 840844.

[5] Diemling, K., Nonlinear functional analysis, Springer-Verlag, New York and Berlin, 1985.

[6] Gaur, A.K. and Mursaleen, Difference in Sequence spaces, Internat. J. Math. and Math. Sci. 21 (4) (1998), 701-706.

[7] Fricke, G.H. and Fridy, J.A., Matrix summability of geometrically dominated series, Canad. J. Math. 39 (1987), 568-582.

[8] Fricke, G.H. and Fridy, Sequence transformations that guarantee a given rate of convergence, Pacific J. Math. 146 (1990) 239-246.
[9] Keagy, T.A. and Ford,W.F., Acceleration by subsequence transformations, Pacific J.Math. 132 (1988), 357-362.

[10] Kizmaz, H., On certain sequence spaces, Canadian Math. Bull. 24 (2) (1981), 169-176.

[11] Lascarides, C.G. and Maddox, I.J., Matrix transformations between some classes of sequences, Proc. Camb. Phils. Soc. 68 (1970), 99-104.

[12] Maddox, I.J., Continuous and Köethe Toeplitz duals of certain sequence spaces, Proc.Camb. Phil. Soc. 65 (1969), 431-435.

[13] Malkowsky, E., A note on Köthe Toeplitz duals of some generalized sets of bounded and convergent difference sequences, J. Analysis 3 (1995).

14. Malkowsky, E., Absolute and ordinary Köthe -Toeplitz duals of some sets of sequences and matrix transformations, Publ. Inst. Math. ( Beograd ), 46 (60) (1989), 97-103.

[15] Mishra, S.K., Sequence space and Related Topics, Ph.D.Thesis, IIT / Delhi (1993).

[16] Mursaleen, Gaur, A.K. and Saif, A.H., Some new sequence spaces, Their Duals and Transformations, Italian Journal of Pure and Applied Mathematics 4 (1998), 127-132.

[17] Peyerimhoff, A., Über ein Lemma von Hern Chow, J. London Math. Soc. 32 (1957), 33-36.

[18] Sarigöl, M.A., "On difference sequence spaces, J.Karandeniz Techn. University”, Fac. Art. Sci. Ser. Math. Phys. 10 (1987) (63-71)

[19] Simons, S., The sequence spaces $\lambda\left(p_{v}\right)$ and $m\left(p_{v}\right)$, Proc. London Math. Soc.15 (3) (1965), 422-436. 
